\title{
Promoting Health
}

\section{Stewart Piper}

By introducing the theories and practices of health promotion, emergency nurses can become strategic practitioners.

Historically, health promotion has been regarded as a marginal hospital activity (Tones and Tilford 2001). But, as the concept of health promotion has changed from one that primarily focuses on individuals (Dooris 2005, Whitelaw et al 2001) to one that includes populations, this view has changed (Dooris and Hunter 2007, Johnson 2000, Johnson and Baum 2001).

Although patients are often unable to take control of their own health needs when in hospital, organisational and environmental changes can help to improve their health (World Health Organization (WHO) 1991, 1997).

For these changes to occur, hospitals, and emergency departments, must move beyond a narrow, medical model agenda (Hancock 1999) and must reject hierarchical forms of management.

Instead, they must embrace inclusive and transparent forms of decision making that involve nurses and patient representatives (Dooris and Hunter 2007, WHO 1991, 1997).

They should also incorporate the theory, values and standards of health promotion into the planning, development and delivery of health care.

This article outlines the concept of health promotion in hospitals, particularly in emergency departments (EDs), and discusses the need for emergency nurses to act as strategic practitioners. It also reviews the application of clinically focused quality indicators of practice (Groene 2006, Rushmere 2000) concerning patient care, information and assessment.

\section{Health promotion concepts}

The shift in operational management and decision making methods has been driven by the WHO's (1997) health promoting hospitals initiative, which concerns:

- Improving the quality of hospital environments, and of catering and hotel services for patients, visitors and staff.

- Minimising health risks.

- Empowering patients, and ensuring that their cultural needs are met and that they are treated with dignity and equity of care.

- Ensuring patient access to good quality health information.

- Improving nurse-patient and interprofessional communication.

- Ensuring that professional and lay education and skills training programmes are provided.

- Ensuring that there is collaboration between health and social care services, support groups and other community-based organisations.

- Ensuring that hospitals focus on public health, including surveillance and monitoring diseases and injuries, when devising of policies and illness-prevention strategies.

The concept of health promotion is open to interpretation. Whitelaw et al (2001) propose five forms of health promotion in hospitals:

- 'Passive' health promotion, where hospitals are simply the settings for attempts to influence the lifestyles of patients generally.

- 'Active' health promotion, where hospital policies promote interventions and outcomes that focus on individual patients.

- 'Vehicle' health promotion, where promoting health becomes a vehicle for hospitals to change their policies and structures. 
- 'Organic' health promotion, where hospitals' contribution to health problems, is acknowledged, but where solutions for individual patients are also provided.

- 'Comprehensive' or 'structural' health promotion, where hospitals contribute to health problems, and change their policies and structures to solve these.

While health promoting hospitals are concerned with the health care of whole patient populations, staff on wards and in EDs are concerned with health promotion among patient groups.

Primary prevention, such as the use of leaflets and videos in waiting rooms to raise awareness among patients, and their relatives and friends, of the risks of smoking-related illnesses, for example, is an important part of the role of EDs (Bensberg and Kennedy 2002, Piper 1999).

But, as this year's RCN Emergency Care Association conference, in Cheshire, revealed, emergency nurses want their voice to be heard outside, as well as inside, the ED.

To this end, emergency nurses can, with the help of professional organisations, such as the $\mathrm{RCN}$ and local inter-agency forums, speak out about health inequalities and deprivation, the advertising of health-damaging products, and workplace health and safety issues. Or they can contribute to initiatives such as the United Nations' Global Road Safety Week, as the editorial in last month's Emergency Nurse (Picton 2008) made clear.

In addition to these politicised approaches, however, there is an ED health promotion agenda that concerns quality and standards of practice, and the management of resources to maximise clinical outcomes.

This agenda is important because, in an evidence-based culture, all healthcare interventions must be research driven and subject to health impact assessments to demonstrate that they deliver the best health outcomes. This agenda involves:

- Professional and interprofessional education and training programmes to provide consistently good care

- Improving skill mix and deploying senior clinical staff to best effect so that each patient is treated by the professional with the best skills to meet their needs' (Jones 2008).

- Designing patient-centred services(Jones 2008).

- Using evidence-based advanced life support, disease management protocols and national service frameworks.

- Ensuring that patient care is collaborative.

- Establishing and measuring the performance of trauma teams by critical incident analyses and the use of trauma scores.

- Ensuring compliance with minimum waiting time operational standards.

\section{Strategic agenda}

In attempting to promote health according to a strategic agenda such as this one, emergency nurses must also consider patient empowerment. This is concerned with shifting the balance of power in health care away from nurses and toward patients so that:

- The maximum amount of healthcare information is made available.

- Equality of service access is ensured, for example by using interpreters.

- Patient autonomy and their decisions on health care are respected.

- Patients can make complaints and obtain fair redress for grievances by using patient advocates and champions.

- Patients can participate in the planning of service developments, the setting of health standards, and the monitoring and evaluation of healthcare services (NHS Management Executive 1993). 
Nurses, as the largest group of healthcare professionals in the UK, are ideally placed to spearhead a range of direct health promotion initiatives.

Yet, the performance of the profession in promoting health has been disappointing (Whitehead 2005).

One area in which emergency nurses can redress this situation is by using quality indicators and standards of health promotion that focus on customer care (Rushmere 2000), patient information and patient assessments (Groene 2006).

In using these indicators, emergency nurses must act as strategic practitioners. They should involve patients in decisions about their health care, ensure that patients know how to complain if they are dissatisfied with the care they have received, and enable patients to make informed choices when asked to participate in clinical research and student training programmes.

Other quality indicators concern patient access to telephones and refreshments, as well as catering and other facilities that are appropriate for patients from a variaty of ethnic backgrounds and those with special needs (Rushmere 2000).

The standards concern disease or injury aetiology, diagnosis and prognosis, as well as patient rehabilitation and sources of support.

When acting as strategic practitioners, emergency nurses are not involved in one-to-one health promoting interventions, but in ensuring that health promotion is a central feature of healthcare practice. Their success can be measured by checking ED notes for the number of patients, expressed as a percentage of the total, who on any one day have been educated in, or informed about, for example, how to manage their injuries or diseases, and how to modify risk factors(Groene 2006).

Groene's (2006) auditable indicators also concern lifestyle risk factors such as smoking, alcohol intake and diet, as well as long-term diseases, discharge planning and sensitivity to sociocultural needs.

The success of emergency nurses as strategic practitioners can also be measured by assessing levels of patient satisfaction or the amounts of information on support groups given to patients.

Tones and Tilford (2001) support the contention that the process and outcomes of health promotion should be recorded in patients' notes and that prospective health promotion needs should be identified when planning care.

\section{Conclusion}

Emergency nurses have a specific role to play in promoting health, not only by taking part in one-to-one nurse-patient interventions, but also by becoming involved in organisational and policy based practice, quality initiatives and associated strategies and policy development. In doing so, they can become strategic practitioners.

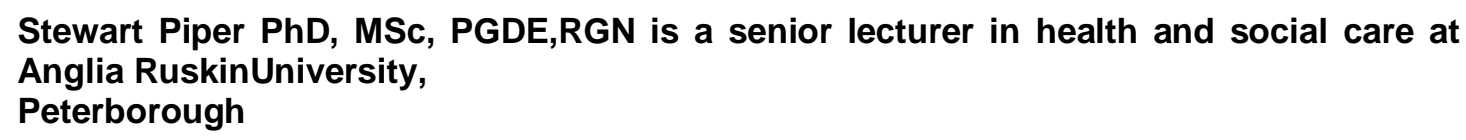

Stewart Piper PhD, MSc, PGDE,RGN is a senior lecturer in health and social care at Anglia RuskinUniversity, Peterborough 


\section{References}

Bensberg M, Kennedy M (2002) A framework for health promoting emergency departments. Health Promotion International. 17, 2, 179-188.

Dooris M (2005) Healthy settings: challenges to generating evidence of effectiveness. Health Promotion International. 21, 1, 55-65.

Dooris M, Hunter DJ (2007) Organisations and settings for promoting public health. In Lloyd CE, Handsley S, Douglas J et al (Eds) Policy and Practice in Promoting Public Health. Sage, London.

Groene $\mathbf{O}$ (2006) Implementing Health and Promotion in Hospitals: Manual and self $\square$ assessment forms. World Health Organization Europe, Copenhagen.

Hancock T (1999) Creating health and health promoting hospitals: a worthy challenge for the twenty-first century. International Journal of Health Care Quality Assurance. 12, 2, 819.

Johnson JL (2000) The health care institution as a setting for health promotion. In Poland BD, Green LW, Rootman I (Eds) Settings for Health Promotion: Linking theory and practice. Sage, London.

Johnson A, Baum F (2001) Health promoting hospitals: a typology of different organisational approaches to health promotion. Health Promotion International. 16, 3, 281-287.

Jones G (2008) Introduction: nursing in emergency care. In Dolan B, Holt L (Eds) Accident and Emergency: Theory into practice. Baillière Tindall, London.

NHS Management Executive (1993) Patient Empowerment. NHSME, London. Picton C (2008) Road safety is no accident. Emergency Nurse. 16, 7, 1.

Piper SM (1999) Health promotion and accident and emergency nursing: theory and practice. In Dolan B, Holt L (Eds) Accident and Emergency Care: Theory and practice. Baillière Tindall, London.

Rushmere A (2000) Health Promoting Hospitals and Trusts: Self assessment and peer review toolkit. Wessex Institute for Health Research and Development, Southampton.

Tones K, Tilford S (2001) Health Promotion: Effectiveness, efficiency and equity. Nelson Thornes, London.

Whitehead D (2005) Health promoting hospitals: the role and function of nursing. Journal of Clinical Nursing. 14, 11, 20-27.

Whitelaw S, Baxendale A, Bryce C et al (2001) 'Settings' based health promotion: a review. Health Promotion International. 16, 4, 339-353.

World Health Organization (1991) The Budapest Declaration on Health Promoting Hospitals. www.euro.who.int/document/ihb/hphbudadecl.pdf (Last accessed: November 25 2008.)

World Health Organization (1997) The Vienna Recommendations on Health Promoting Hospitals. www.natverket-hfs.se/dokument/6.pdf (Last accessed: November 25 2008.) 